\title{
BPM SYSTEM FOR THE SWISS LIGHT SOURCE
}

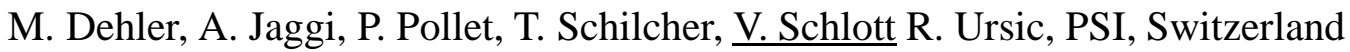

\begin{abstract}
The layout of the SLS beam position monitor (BPM) system is presented. Since sub-micron position data in normal closed orbit and feedback mode as well as turn by turn information are required, the SLS BPM electronics pursues a new digital approach. The self calibrating four channel system consists of a RF front end, a digital receiver and a DSP controller. The whole system is integrated in the EPICS control system, which allows to select between different operation modes, so that the same BPM electronics applies to all the sections of the machine, namely linac, transfer lines, booster and storage ring. Mechanical drifts will be monitored by an independent measurement system and taken into account, when processing the final electron beam position.
\end{abstract}

\section{INTRODUCTION}

One of the challenges of the SLS project is to ensure the beam quality needed for the production of high brilliance synchrotron radiation requiring a wide range of beam diagnostics measurements. Therefore, the beam position monitor system has to provide a variety of operation modes.

One extreme (in terms of requirements) is the mode used for the closed orbit feedback. Sine the feedback has to reduce the electron beam jitter to less than $\sigma / 10$ of the vertical beam size in the ID sections, the corresponding measurements require sub micron precision at a bandwidth of a few $\mathrm{kHz}$. The other extreme is to take a snap shot of the beam orbit in turn-by-turn mode, a high speed measurement requiring an bandwidth of about $0.5 \mathrm{MHz}$. An overview of the technical specifications and the measurement modes is given in table 1 .

\section{MECHANICAL ALIGNMENT}

At SLS, the BPM stations, six per ring sector (layout see figure 1), are rigidly attached to the girders and serve as supports for the vacuum system. The mechanical prealignment gives a relative position deviation of the BPM center with respect to the adjacent quadrupole axis of less than $\pm 25 \mu \mathrm{m}$. A more precise alignment to less than $10 \mu \mathrm{m}$ can subsequently be obtained with the stored electron beam, applying the method of beam based alignment BBA [1]. A complete BBA cycle will take about 15 minutes and will bes performed after every start-up of the machine.

During operation, thermal effects lead to mechanical drifts of the BPM chamber, which have been simulated to be in the order of $2 \mu \mathrm{m} / \mathrm{K}$ for SLS. This is already critical for the transverse closed orbit feedback system. Therefore, the mechanical drifts of the BPMs with respect to
Table 1: Digital BPM system specifications.

\begin{tabular}{|l|r|}
\hline Parameter & Specification \\
\hline Dynamic range by mode: & \\
Multi Bunch & $1-500 \mathrm{~mA}$ (avg.) \\
Single Bunch & $1-20 \mathrm{~mA}$ (avg.) \\
Pulsed and First Turn & $1-10 \mathrm{~mA}$ (peak) \\
\hline Position Measuring Radius & $5 \mathrm{~mm}$ \\
\hline Resolution (2 kHz bandwidth) & $<1 \mu \mathrm{m}$ \\
\hline Beam Current Dependence & $<100 \mu \mathrm{m}$ \\
$1-400 \mathrm{~mA}$ & $<5 \mu \mathrm{m}$ \\
1 to 5 relative & $500 \mathrm{MHz}$ \\
\hline RF and IF frequencies & $36 \mathrm{MHz}$ \\
Carrier RF & $498.5 \mathrm{MHz}$ \\
Carrier IF & $34.5 \mathrm{MHz}$ \\
Pilot RF & $\mathrm{f}_{\text {revolution }}$ \\
Pilot IF & Pulsed \\
\hline Max. data & Booster \\
acquisition rate & Turn-by-turn \\
\hline Feedback mode throughput & Tune \\
\hline Modes of operation & Closed orbit \\
\hline
\end{tabular}

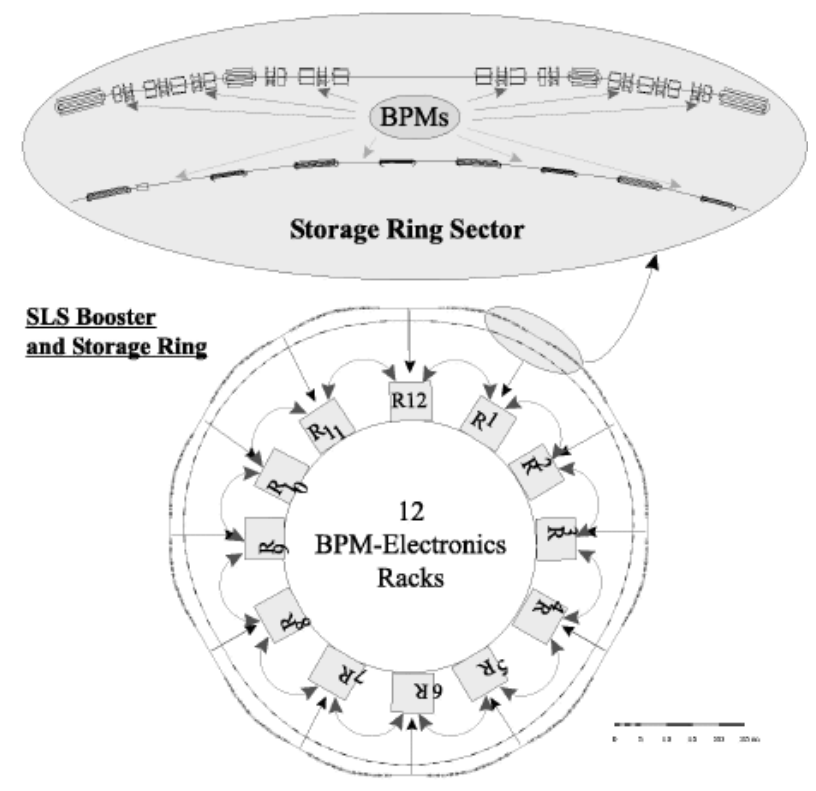

Figure 1: BPM positions including layout of the electronics and feedback architecture. 


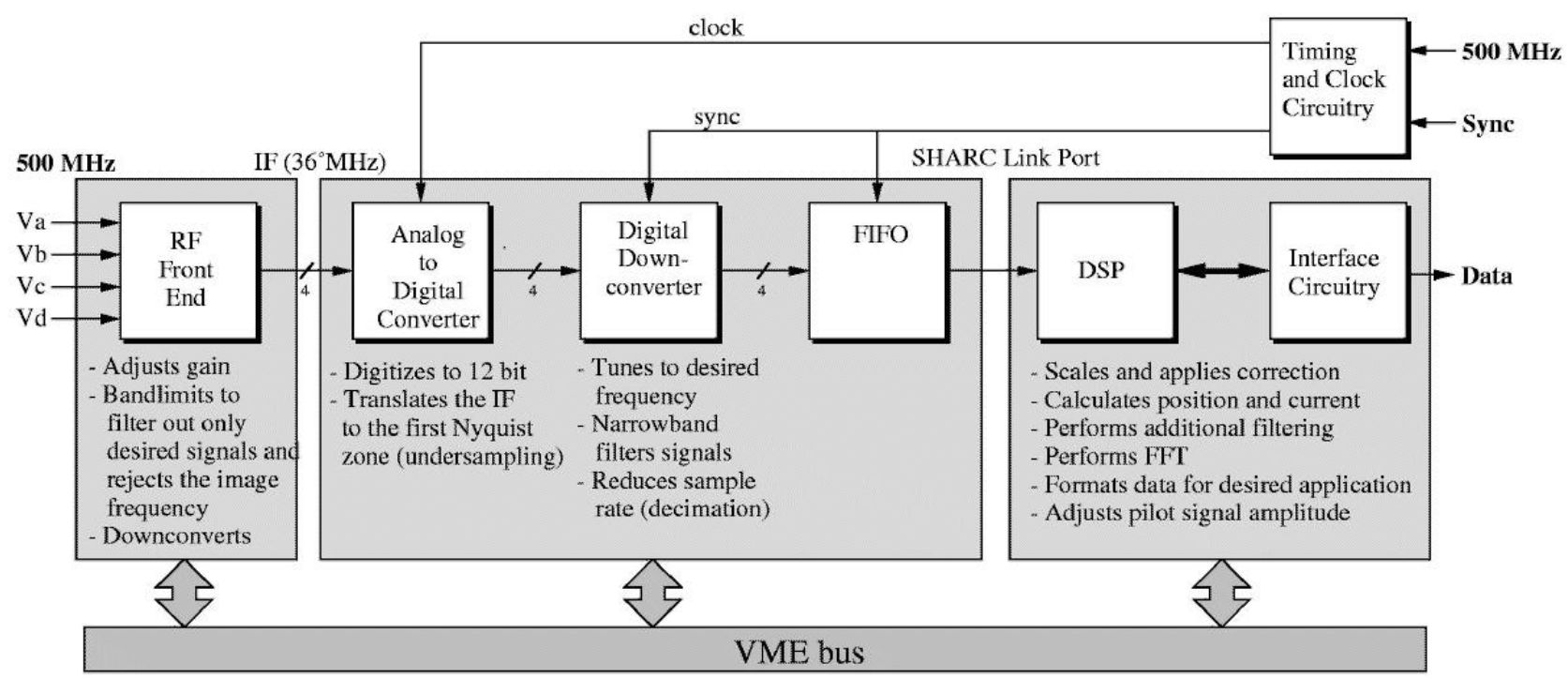

Figure 2: Block diagram of the digital BPM electronics

the adjacent quadrupole magnets are monitored via a set of absolute linear encoders, which are rigidly clamped to the quadrupoles. The system resolution is less than $1 \mu \mathrm{m}$ within a measuring range of $\pm 2.5 \mathrm{~mm}$. The position information from the encoders is continuously recorded by the control system and used to correct the BPM readings.

As a position sensor, the most promising candidate is a linear encoder, which has been jointly developed by former PSI Zürich [2] and Baumer Electric AG [3]. It was originally developed for use in machining tools, handling systems and robotics and offers the required resolution and range. Preliminary tests are currently under way at the SLS test stand as well as at Sinchrotrone Trieste (ELETTRA) in order to examine radiation resistance and to validate the measurement principle.

\section{BPM ELECTRONICS}

The SLS BPM electronics represents a departure from conventional BPM system design insofar, as the same setup delivers high speed/medium precision and low to medium speed/high precision measurements.

The electronics is a four-channel system. The button signals enter the RF front end, where they get mixed to the intermediate frequency of $36 \mathrm{MHz}$ and then pass through a low cost $5 \mathrm{MHz}$ wide Surface Acoustic Wave (SAW) filter. At the input of the digital receiver, the signals are then sampled at $31.25 \mathrm{MHz}$ in case of Storage Ring BPMs (27.78 $\mathrm{MHz}$ in case of Booster BPMs). This means that the 36 $\mathrm{MHz}$ signal, which lies in the third Nyquist zone, is aliased down to $4.75 \mathrm{MHz}(8.22 \mathrm{MHz})$. More about this under sampling concept can be found in [4]. For the A/D conversion we selected the AD9042 analog-to-digital converter (ADC) available from Analog Devices. It is a 12 bit ADC which has $41 \mathrm{MHz}$ maximum sampling rate and $100 \mathrm{MHz}$ analog bandwidth. The remainder of the processing is done in a digital way. Four digital down converters (DDC) first translate the signals to baseband and then filter and deci- mate the data streams. The latter two processes are very important: filtering defines system bandwidth which in turn affects measurement fluctuation; decimation, a reduction of the output data rate with respect to the input, significantly reduces the downstream digital signal processing requirements. We have selected HSP50214 DDC integrated circuit from Harris. It is one of the more sophisticated and flexible programmable down converters available on the market today. In the last stage, digital signal processors (DSP) calculate the beam positions, apply correction factors and do further post processing like FFTs. The second task for the DSPs is the real time calculation of corrector settings via the singular value decomposition algorithm, which is used for the closed orbit feedback loop. To that effect, additional communication links to DSPs in the adjacent storage ring sectors are provided [5].

The problem associated with drifting gains of one channel with respect to the others is avoided by a separate pilot signal with a frequency that is $1.5 \mathrm{MHz}$ lower than the RF. Four pilot signals are added to the inputs that are later on demodulated in the DDC and used to keep the gain of the four RF/IF channels matched within the dynamic range. The problem of nonlinearities associated with demodulation is avoided by direct IF sampling. Since all the processing downstream the ADC is done digitally in a DDC, it is the ADC that defines the nonlinearity. The specification for the AD9042 integrated circuit is:

Differential non-linearity : $\pm 0.30 \mathrm{LSB}$ or $\pm 7.3 \cdot 10^{-5} \mathrm{FS}$ Integral non-linearity : $\pm 0.75 \mathrm{LSB} \quad \pm 1.8 \cdot 10^{-4} \mathrm{FS}$

The effective resolution of the complete system is determined by the following. The analog to digital conversion using a 12 bit ADC in itself would restrict the resolution to an order of $3 \mu \mathrm{m}$. The subsequent reduction in measurement fluctuation is due to an improvement in the signal-tonoise ratio gained through the oversampling (with respect to the band of interest) and digital filtering. It is the ratio 
of the passband to the Nyquist bandwidth expressed in $\mathrm{dB}$. This process recovers the missing precision such, that for beam current above $50 \mathrm{~mA}$ and the output sample rate of 4 $\mathrm{kHz}$, which is critical for the closed orbit feedback, beam movements smaller than $0.1 \mu \mathrm{m}$ can be measured. A graph of expected resolution versus current and sampling rate is shown in figure 3.

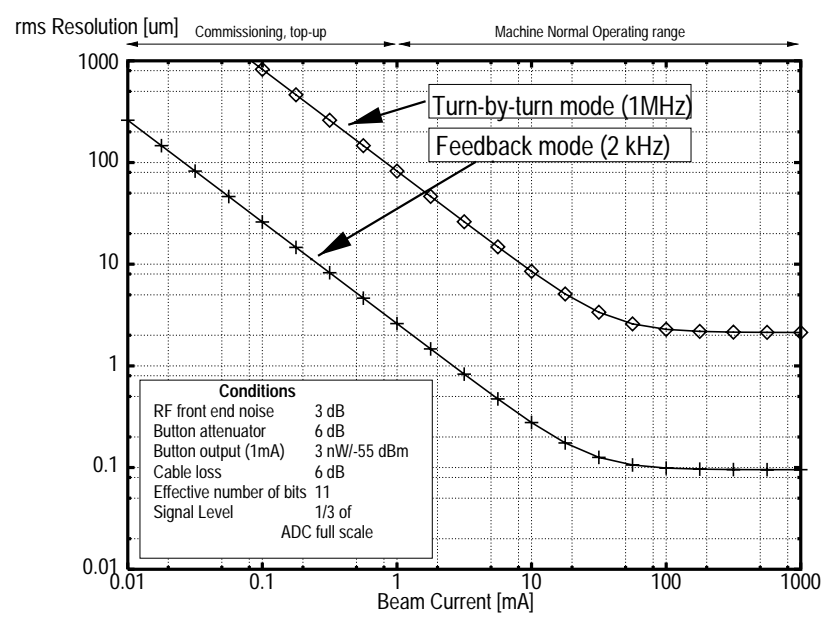

Figure 3: BPM resolution versus current for different operation modes and bandwidths.

\section{CONCLUSION}

For the SLS beam position monitoring system, a wide range of measurement modes, from high speed/medium resolution to low speed/high resolution, is required. To cover these, only one type of system will be used, which provides the flexibility to adapt to different measurement types by the use of programmable digital down converters. The DSP back end allows further high level post processing like FFT and an implementation of the closed orbit feedback algorithm as an integral part of the BPM electronics. Compared to more conventional solutions, the approach is cost advantageous, it offers the required sub micron resolution and programmable bandwidth, including turn-by-turn capability at a cost lower than that of two separate electronic systems to perform the same set of functions. As a nearly free feature, the closed orbit feedback is integrated into the BPM system.

The BPM stations are going to be prealigned with respect to the adjacent quadrupole magnet. This setting are updated and refined at each new run using the method of beam based alignment. Drifts occurring during operation will be monitored by absolute linear encoders, whose readings are used to recalibrate the BPM center position.

An evaluation of the electronic system using an experimental setup of commercial ADC, digital down converter and digital signal processor boards very similar to the proposed system has shown the validity of the approach. A first prototype is in work, first tests with beam are planned in the first half of this year at the synchrotron light facility
ELETTRA in Trieste/Italy.

\section{ACKNOWLEDGEMENTS}

We would like to thank the controls and instrumentation groups at the synchrotron light facility ELETTRA/Italy for the support in a fledgling collaboration and for giving us the possibility for various tests using their accelerator facility.

\section{REFERENCES}

[1] P. Röjsel, "BPM System for Storage Rings, Measuring Beam Position against Quadrupole Magnets Magnetic Center", proc. EPAC'94, pp 1557, (1994)

[2] K. Engelhard and P. Seitz, "Absolute High-Resolution Optical Position Encoder”, Appl. Optics, Vol. 35, No.1, 201-208, (1996).

[3] LHKK 016B03S01.XXX, "Absolute Position Encoders", Baumer Electric AG, Frauenfeld, Switzerland.

[4] R. Ursic, "Digital receivers offer new solutions for beam instrumentation", this conference.

[5] M. Boege, M. Dehler, T. Schilcher, V. Schlott, R. Ursic, "Fast Closed Orbit Control in the SLS Storage Ring", this conference. 